\title{
Optimized Analysis on the Working Process of Engine Variable Gas Distribution Phase
}

\author{
Wang Fengjun \\ Wuxi Institute of Commerce, Wuxi, Jiangsu 214153, china
}

Keywords: Engine; Valve timing mechanism; Variable gas distribution phase

\begin{abstract}
In this paper, a calculation model of 491Q gasoline engine working process was established using the engine one-dimensional simulation software BOOST, and through the analysis on the curve of pressure fluctuation in the front of the inlet valve at the speeds, the relationship between the late closed angle of the inlet valve and the performance parameters of the engine was concluded, and the late closed angle of the inlet valve on the basis of the principle of camshaft phase modulation was determined and optimized. After the simulated calculation of the engine adopting the optimized late closed angle of the inlet valve was done again, the results show that when the late closed angle of the inlet valve on the engine lagged by $10^{\circ} \mathrm{CA}$, the maximum power increased by $3.6 \%$, the power and torque in the high speed zone were on average increased by $3 \%$ $7 \%$, and the fuel economy was also much improved.
\end{abstract}

\section{Introduction}

Among the domestic engines, 491Q gasoline engine has good economic and social benefits. However, with the increasingly stringent emission regulations, and the higher requirements of people for engine performance and economy, it can no longer meet the needs of the society. But the model has still the potential of further improvement, and after a resonant intake system is adopted for the model, the charging coefficient of the engine was almost greatly improved in the whole speed range, especially improved more significantly in the medium speed range. Nonetheless, the charging coefficient is still very low in the high speed range, and the high speed performance of the model failed to be given full play to, while all aspects of the engine performance can also be further improved by further using the variable gas distribution phase technology [1].

\section{Establishment of the Model}

Using BOOST software in this paper, based on the one-dimensional hydrodynamics, numerical calculation of the piping system was done with the adoption of finite volume method, and full consideration was given to the changes in the corresponding thermodynamic properties caused by different components of the combustible mixture. Moreover, many different types of boundary condition submodels were provided to choose, and simulated calculation of various forms of the engine's whole or partial subsystems could be carried out [2][3]. The whole software consists of pretreatment module, main calculation module and pretreatment module. BOOST pretreatment module is the corresponding model established by adopting a modular structure, the engine's components (such as cylinder, air filter, pipe joint and cavity volume) are expressed as the corresponding modules, and they are connected with line-oriented pipe. These modules only have a symbolic meaning, and all structure parameters and characteristics parameters of the actual specific engine need to be defined in the data input window of corresponding elements of the total input window. The calculation results can analyzed in a variety of forms in the pretreatment module.

In this paper, the whole simulation model of 491Q gasoline engine was established by adopting the resonant intake system, as shown in Figure 1.

The model is mainly composed of cylinder, air intake system and exhaust system. The air intake system mainly includes air filter F1, resonant cavity Pl1, intake manifold tube 1-8 and resonant tube 14 and other modules. The exhaust system is mainly composed of exhaust manifold tubes 9-12 and 15-22, branch pipe joints J1, J2 and J3, main pipe 23, flow constraint 
R5 and muffler Pl2, etc. A catalyst in the model was simplified to a flow constraint, and the branch pipe joints were processed with the Benson's momentum model. A relatively simple single area Weber function was adopted as combustion model in the cylinder, and the empirical formula proposed by Wossini in 1978 was chosen as the heat exchange coefficient.

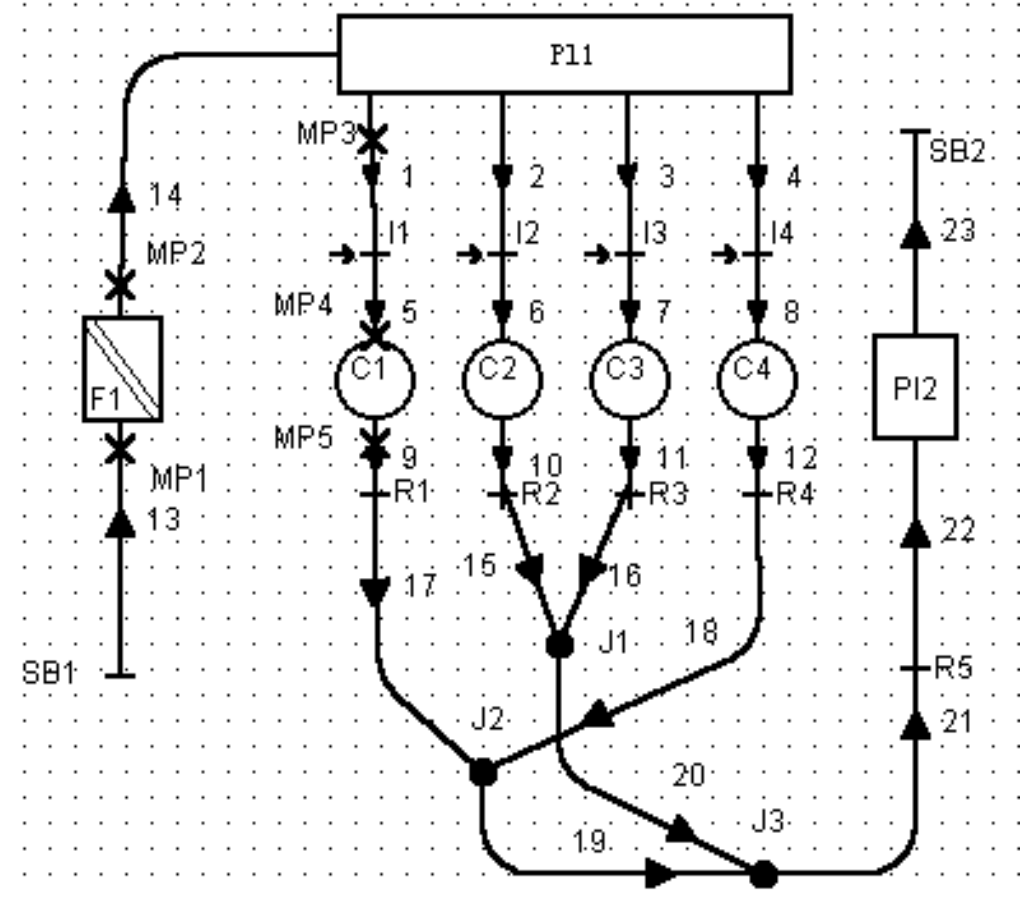

Fig. 1 Simplified engine model

In addition, legend in the model C1-C4 said cylinder, SB1, SB2 said boundary, MP1 MP5 for measurement point. The corresponding basic data of the engine are shown in Table 1 and Table 2 .

Table 1 Major parameters of the engine

\begin{tabular}{c|c|c|c|c|c}
\hline Number of cylinders & 4 & Mode of fuel supply & $\begin{array}{c}\text { Multi-point } \\
\text { injection }\end{array}$ & $\begin{array}{c}\text { Angle of combustion } \\
\text { duration }\left(^{\circ}\right)\end{array}$ & 50 \\
\hline Stroke number & 4 & $\begin{array}{c}\text { Length of connecting rod } \\
(\mathrm{m})\end{array}$ & 0.12975 & $\begin{array}{c}\text { Advance angle of } \\
\text { inlet valve }\left(^{\circ}\right)\end{array}$ & 15 \\
\hline $\begin{array}{c}\text { Number of valves on } \\
\text { each cylinder }\end{array}$ & 2 & Ignition sequence & $1-3-4-2$ & $\begin{array}{c}\text { Late closed angle of } \\
\text { inlet valve }\left(^{\circ}\right)\end{array}$ & 53 \\
\hline $\begin{array}{c}\text { Compression ratio } \\
\text { Displacement (L) }\end{array}$ & 2.237 & $\begin{array}{c}\text { Atmospheric temperature } \\
(\mathrm{K})\end{array}$ & 298 & $\begin{array}{c}\text { Advance angle of } \\
\text { exhaust valve }\left(^{\circ}\right)\end{array}$ & 53 \\
\hline $\begin{array}{c}\text { Cylinder diameter } \\
(\mathrm{m})\end{array}$ & 0.091 & $\begin{array}{c}\text { Diameter of inlet valve } \\
(\mathrm{m})\end{array}$ & 0.031 & $\begin{array}{c}\text { Late closed angle of } \\
\text { exhaust valve }\left({ }^{\circ}\right)\end{array}$ & 13 \\
\hline $\begin{array}{c}\text { Stroke (m) } \\
\text { Am }\end{array}$ & 0.086 & $\begin{array}{c}\text { Diameter of exhaust valve } \\
(\mathrm{m})\end{array}$ & 0.031 & - & - \\
\hline
\end{tabular}

Table 2 Basic data of piping system

\begin{tabular}{c|c|c|c|c|c|c|c|c}
\hline Pipe No. & Pipe length & Diameter & Pipe No. & Pipe length & Diameter & Pipe No. & Pipe length & Diameter \\
\hline 1 & 0.4 & 0.03 & 9 & 0.08 & 0.032 & 17 & 0.305 & 0.032 \\
\hline 2 & 0.4 & 0.03 & 10 & 0.08 & 0.032 & 18 & 0.285 & 0.032 \\
\hline 3 & 0.4 & 0.03 & 11 & 0.08 & 0.032 & 19 & 0.36 & 0.037 \\
\hline 4 & 0.4 & 0.03 & 12 & 0.08 & 0.032 & 20 & 0.29 & 00.037 \\
\hline 5 & 0.01 & 0.0335 & 13 & 0.11 & 0.05 & 21 & 0.1 & 0.035 \\
\hline 6 & 0.01 & 0.0335 & 14 & 0.35 & 0.08 & 22 & 0.25 & 0.04 \\
\hline 7 & 0.01 & 0.0335 & 15 & 0.3 & 0.032 & 23 & 0.2 & 0.04 \\
\hline 8 & 0.01 & 0.0335 & 16 & 0.3 & 0.032 & - & - & - \\
\hline
\end{tabular}

\section{Calculation Results and Analysis of Inlet Pressure on the Engine}

The pressure on the engine inlet valve has a decisive influence on the charging efficiency of the 
engine, and the charging efficiency of the engine is decided by it jointly with the pressure inside the cylinder and the flow area of the inlet valve. Therefore, the variable gas distribution phase system based on the principle of camshaft phase modulation was designed to make the inlet valve closing angle adapt to the pressure waveform of the inlet valve, so that it was nearly close to pressure peak value when the inlet valve was closed, in order to reach the maximum charging efficiency.

Engine Speed near the Low-order Resonant Speed. According to the waveform data of pressure on the cylinder 1 inlet valve of the engine at different rotational speeds, when the engine speed is near the low-order resonant speed, the inlet valve pressure shows a regular periodic wave. When the inlet valve is open, the pressure wave is up to peak, and since then, the inlet valve pressure drops sharply, and it will reach the peak again after the inlet valve is opened by about $180^{\circ} \mathrm{CA}$. This is because after the inlet valve is open, the engine is in the intake stroke, and the piston moves quickly to the lower dead center from the upper dead center [4]. At this time, the cylinder pressure drops rapidly, and a large expansion wave is formed at the inlet valve to lower down the inlet pressure, while air starts to flow from airway through the inlet valve into the cylinder. The expansion wave flows along the intake branch, resonant cavity and resonant tube to the resonant nozzle, and reflects to be compression wave on the resonant tube, causing the pressure fluctuations inside the resonant intake system. In the work cycle of the engine, each cylinder excites pressure waves during the opening process of the inlet valve, and they interact in the resonant cavity and the resonant tube. The pressure waves with consistent phases are generated in the resonant intake system, and the pressure is reflected back to the inlet valve of each cylinder in the engine. When the engine speed gets closer to the low order resonant speed, the pressure amplitude value of the inlet valve is bigger, and the pressure peak value is closer to the opening moment of the inlet valve. When the engine speed is lower than the low-order resonant speed, the pressure waves of the inlet valve move forward. When the engine speed is higher than low-order resonant speed, the pressure waves of the inlet valve move backward.

Engine Speed far from Low-order Resonant Speed. When the engine speed is far from low-order resonant speed, the low order resonance effect has little impact on the inlet valve pressure.in the high speed zone, the pressure waveforms are basically the same, but the periodic pressure waves are damaged. When the inlet valve is open, the inlet valve pressure drops sharply, becoming a "valley", and then the pressure waves begin to rise and are up to peak before the inlet valve is closed. This is because after the inlet valve is opened, the engine enters into the intake stroke, and the pressure in the cylinder drops, forming a negative pressure wave in the inlet valve. In the second half of the intake stroke, as the piston downward speed slows down, the opening area of the inlet valve decreases, and the pressure inside the cylinder rises gradually.in addition, the kinetic energy of the gas in the intake port will translate into pressure, so as to make the inlet valve pressure rise and become peak before the inlet valve is closed. The higher the engine speed, the higher the pressure fluctuation amplitude, this is because the higher the speed, the bigger the gas flow rate, and the influence of kinetic energy converted into pressure fluctuation is more obvious.

Engine Speed close to High-order Resonant Speed. However, when the engine speed is close to the high order resonant speed, the expansion wave generated by opening the inlet valve is reflected as a compression wave when reaching the resonant cavity, and the compression wave returns to the inlet valve along the intake branch, forming a positive pressure wave.at the arrival time of the positive pressure wave, the valve flow area is large, and this is of great help to enhance the charging efficiency of the engine. When the engine speed is lower than the high order resonant speed, the pressure wave phase moves forward, and the pressure amplitude decreases and the charging efficiency drops at this time. When the engine speed is higher than the high order resonant speed, the pressure wave phase moves backward, and the pressure shows a deep "trough" at this time and the peak is present after the inlet valve is turned off, while the charging efficiency falls sharply [5].

It can be obtained through the above analysis that the crankshaft angle corresponding to the pressure peak in the front of the inlet valve of the cylinder 1 in medium and high speed zone is 
shown in Table 3.

Table 3 Crankshaft angle of pressure peak

\begin{tabular}{c|c|c|c|c|c|c}
\hline Engine speed r/min & 3000 & 3500 & 4000 & 4500 & 5000 & 5500 \\
\hline Peak crank angle $\left(^{\circ}\right)$ & 565 & 575 & 580.5 & 610 & 630 & 645 \\
\hline Position of lower dead center $\left(^{\circ}\right)$ & 15 & 35 & 40.5 & 70 & 90 & 105 \\
\hline
\end{tabular}

\section{Identification and Optimization of the Inlet Valve Phase}

Through the inlet valve variable timing analysis, we can know that in the intake stroke, because a positive pressure wave in the intake pipe is generated by the piston downward, the phase of the wave crest relative to the crankshaft angle moves backward away from the upper dead center as the engine speed rises, and the change relations is shown in table 2-1. When the engine ran at a high speed, the inertia supercharging effect of the inlet charging could be made full use of by delaying closing the inlet valve, to improve the charging efficiency of the engine cylinder, so as to improve the dynamic performance and economic performance of the engine. However, because the inertia supercharging effect of inlet charging disappeared at a low rotational speed, in order to ensure the maximum effective compression ratio, the inlet valve was no longer delayed to be closed. After the resonant intake system was implemented in the 491Q engine, due to poor matching with distribution phase in the high-speed stage, serious backflow appeared at the beginning of gas inflow. Starting from 3500r/min speed, the charging efficiency began to decline, and the decline range accelerated with the increase of rotational speed, seriously affecting the machine's high speed performance. In order to improve the charging efficiency of the machine at the high speed, the late closed angle of the inlet valve in the distribution phase was optimized based on the principle of camshaft phase modulation.

Change Relationship of Distribution Phase along with Crank Angle. The principle of camshaft phase modulation shows that when the late closed angle of the inlet valve changes, because of the original cam contour line of the engine remains the same, the early open angle of the inlet valve will change in conjunction with the phase [6]. The change relationship of the inlet and exhaust valve phase with crank angle is shown in Figure 2.

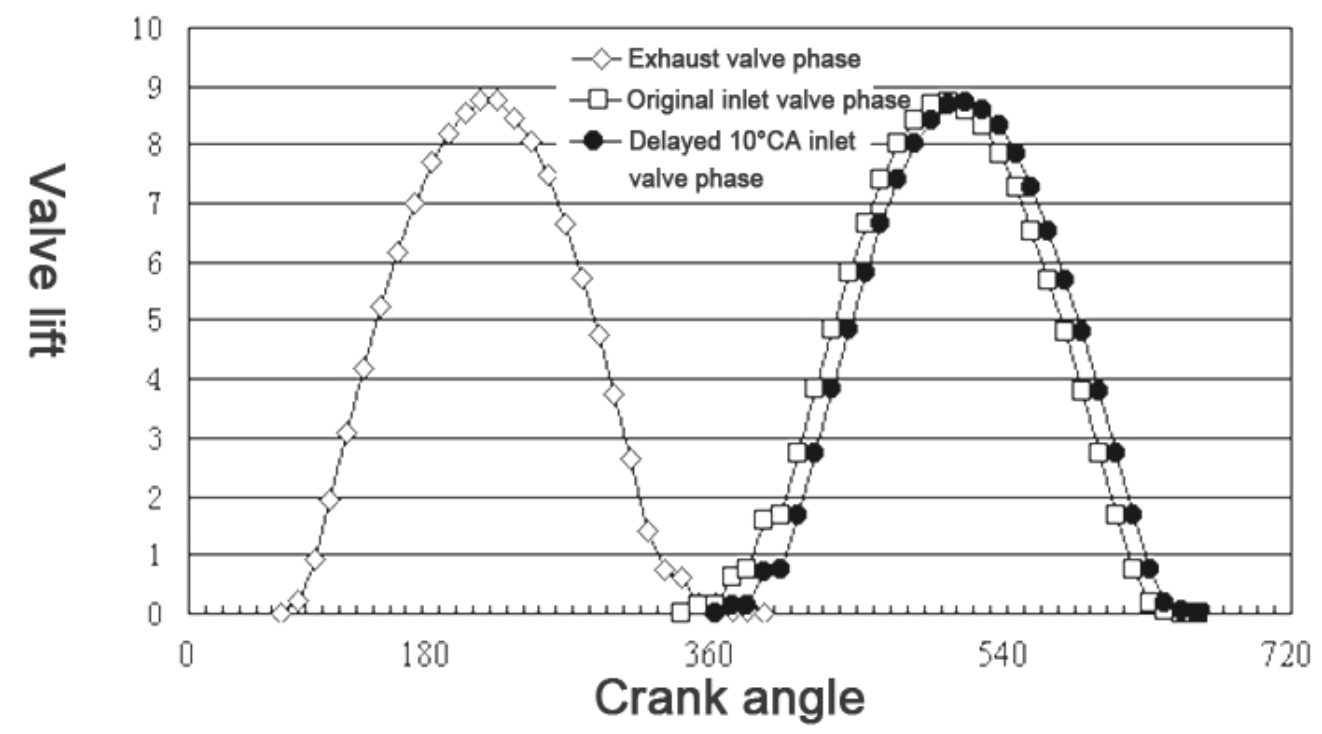

Fig.2 Change in inlet and exhaust valve lift with crank angle

Optimization of the Late Closed Angle of the Inlet Valve. We already know from the analysis above that the late closed angle of the inlet valve and the overlapping angle of the valve in the gas distribution phase of the engine had the greatest influence on the engine performance, and the early opening angle of the inlet valve changed with the same phase along with the late closed angle of the inlet valve, so it would inevitably result in the overlapping angle of the valve to change also. The late closed angle of the inlet valve herein was only optimized analyzed.

Hence, under the condition of other parameters constant, we selected several typical working 
conditions, 1000r/min, 2000r/min, 3000r/min, 4000r/min and 5000r/min, and set the late closed angle of the inlet valve to be variable parameters for simulated calculation. The improvement in high speed power performance of the engine was mainly considered in this article, so we selected the engine torque as the main evaluation index, while considering the charging efficiency and analyzing the engine performance under various operating conditions along with the change trend of the inlet valve's late closed angle.

When the late closed angle of the inlet valve was set as a variable parameter, as the late closed angle of the inlet valve changed, the engine torque slightly decreased at low speeds, but changed little. The torque increased large in the high speed zone, and with the increase in the lagged value of the late closed angle of the inlet valve, the torque values increased also further. But considering that the early opening angle of the original inlet valve of the engine was only $15^{\circ} \mathrm{CA}$, and the late closed angle of the inlet valve would also have a negative impact to other engine performance parameters, so the lagged value of the late closed angle of the inlet valve was limited. Combined with working conditions, it was appropriate to determine that the late closed angle of the inlet valve lagged $10^{\circ} \mathrm{CA}$ on the basis of the original machine (that is, the inlet valve was closed $63^{\circ} \mathrm{CA}$ behind the lower dead center).

Choice of Breaking Point. According to the above analysis, the speed characteristics were simulated and calculated respectively in both cases under which the late closed angle of the inlet valve was original and lagged to be closed by $10^{\circ} \mathrm{CA}$.after the machine was turned off when the phase of the inlet valve lagged by $10^{\circ} \mathrm{CA}$, the maximum power was raised from $84.4 \mathrm{~kW}$ to $87.3 \mathrm{~kW}$, with an average increase of power and torque $3 \%$ to $7 \%$. When the original machine was closed at the maximum torque $3200 \mathrm{r} / \mathrm{min}$ and the phase lag of the inlet valve $10^{\circ} \mathrm{CA}$, the maximum torque was almost not increased, but the high speed torque tended to flat and generally increased. When the inlet valve was lagged to be closed, the power of the engine in the medium and high speed zone was increased and at the same time, the fuel consumption rate was also reduced. Through analysis, the reason is mainly because when the engine was in the medium and high speed zone, the admission camshaft phase lagged, enabling the phase angle for opening the inlet valve to be delayed accordingly. On the basis of the analysis on the impact of variable inlet valve timing on the engine performance, we know that as the inlet valve was late open, the intake flow was sped up at the middle and high speed, which strengthened the intake vortex, increased the combustion rate, obtained a high combustion efficiency, and lowered down the fuel consumption rate of the engine.

Determination of Integral Method. In a word, through the above analysis, we can see that in the gas distribution phase of the engine, the late closed angle of the inlet valve has a great influence on the engine performance. Of which, the late closed angle of the inlet valve can be directly optimized by calculation, it is concluded that the late closed angle of the inlet valve is $63^{\circ} \mathrm{CA}$ behind the lower dead center. However, other parameters of the engine's gas distribution phase also affect the engine performance, especially the valve over-lapping angle. So, if the best performance of the engine is to be achieved, a comprehensive analysis of the phase distribution phase angle must also be done.

Thus, when the engine speed is within the low speed range, the gas distribution phase of the original machine is still used, to make full use of low speed torque characteristics at the original gas distribution phase. After the engine speed exceeds the maximum torque point (3200r/min), an appropriate mechanism can be adopted to rotate the admission cam phase of the valve timing mechanism to the direction of late opening by $10^{\circ} \mathrm{CA}$, in order to make full use of the high power and low fuel consumption rate produced when the inlet valve is closed late.

\section{Conclusion}

In this paper, 419Q engine was simulated to be calculated and analyzed by use of the software BOOST. First of all, a simplified engine model was set up in the pretreatment module, then the changes in pressure waves in front of the 1st cylinder inlet valve of the engine were analyzed, and a lag plan for the inlet valve closing angle on the basis of the principle of camshaft phase modulation 
was determined. Through the analysis, the high speed performance of the engine could be better improved when the inlet valve closing angle lagged by $10^{\circ} \mathrm{CA}$ on the basis of the original machine. The comparison results by simulated calculation show that the variable gas distribution phase has a great influence on the power performance and fuel economy of the engine, and has a great potential to improve the power performance of the engine.

\section{References}

[1] Michael B.Riley, Mark McElwee and Russell Wakeman. A Mechanical Valve System with Variable Lift, Duration, and Phase Using a Moving Pivot. SAE970334.

[2] Hails Peter Lenz, Bernard Gerlnger, Guenter Smetana and Alols Dachs. Inidal Test Results of an E1ectro-Hydraulic Variable-Valve Actuation System on a Firing Engine. SAE890678.

[3] YasuhiroUrata, Hedezo Umiuama, Kluoshi Shimizu, Yoshihiro Fujiyoshi, Hiroshi Sono and Koichi Fukuo. A Study of Vehicle Equipped with Non-Throttling SI Engine with Early Inlet valve Closing Mechanism. SAE930820.

[4] Burak Gecim. Analysis of a Lost-Motion-Type Hydraulic System for Valve Actuation. SAE930822.

[5] Chen Lingshan, Chen Junhua, Chen Boxian. Study and optimized design of variable valve timing for automotive engine. Chinese Internal Combustion Engine Engineering. 2003, (2): 18 to 21.

[6] Wang Tao, Qin De, He Zhiyong. Research of engine variable valve timing technology. Small Internal Combustion Engine and Motorcycle. 2003, (2): 20 to 23. 